\title{
Noninvasive Respiratory Support for the Premature Infant: Choosing the Optimum Interface
}

Preventing lung injury to reduce the burden of bronchopulmonary dysplasia is a crux of neonatal care. We know that decreasing the duration of intubation and duration of mechanical ventilation is a major component of injury prevention. As practices leading to earlier extubation become more common, the use of noninvasive respiratory support to maintain and support respiratory function has increased. It is only natural then, that the number of modes and devices available to deliver this support have increased, which leads to the ever-present question: which one do I choose?

In this issue, Drescher and Hughes ${ }^{1}$ describe their experience with noninvasive respiratory support interfaces and delivery devices. I commend the authors for their efforts to study, analyze, and share the outcome of changing the modality for noninvasive respiratory support in their neonatal ICU because the current body of literature continues to be limited. Currently, modes for noninvasive respiratory support include nasal high-frequency ventilation, noninvasive ventilation (NIV), bi-level positive airway pressure, nasal CPAP, bubble nasal CPAP, high-flow nasal cannula (HFNC), and low-flow nasal cannula. The number of devices that provide this support makes the choices even more numerous, which can become daunting. As the authors point out in this study, one of the benefits to the RAM nasal cannula (Neotech, Valencia, CA) is that multiple modes (though some provided off-label) can be delivered through the same interface which streamlines the delivery of noninvasive respiratory support.

Often in practice, we see that ease of delivery and perceived comfort of noninvasive respiratory support plays an important role in the decision of the modality used. The benefit of nasal CPAP in the preterm infant to provide stable distending pressure is well documented. ${ }^{2-4}$ Nasal

The author has disclosed no conflicts of interest.

Correspondence: Suma B Hoffman MD, Department of Pediatrics, School of Medicine, University of Maryland 110 S Paca Street, 8th Floor Baltimore, MD 21201. E-mail: shoffman@som.umaryland.edu.

University of Maryland, School of Medicine, Department of Pediatrics, 110 S Paca Street, 8th Floor Baltimore, MD 21201. E-mail: shoffman@som.umaryland.edu.

DOI: $10.4187 /$ respcare.06604
CPAP maintains functional residual capacity and reduces work of breathing by stenting the airway, and may promote lung growth. ${ }^{5,6}$ However, providing nasal CPAP with the current approved interfaces can be nursing intensive and cause nasal injury, and is often poorly tolerated by patients. Due to these drawbacks, HFNC has been used as an alternative, given its ease of use, but ease of use does not necessarily lead to improved outcomes.

Although there are several studies that show HFNC to be similar to nasal CPAP, ${ }^{7-9}$ in our own institution, we found a longer duration of respiratory support with the

See the Original Study on Page 1197

implementation of HFNC, ${ }^{10}$ which has been echoed in other studies as well. ${ }^{11,12}$ Despite the results of our study, HFNC continues to be frequently used given the aforementioned drawbacks to the current nasal CPAP interfaces. The RAM cannula, as shown by the authors of the study $^{1}$, has a similar appearance to an HFNC, which would likely have similar perceived comfort and ease of use as HFNC, and, in their study, was noted to cause significantly less nasal breakdown compared with the previous interface used. The ability to provide stable distending pressure through the RAM cannula interface may decrease the desire to wean and/or change support before a patient is truly clinically ready. In addition, the ability to provide NIV via this interface may be beneficial as well. A recent Cochrane review found that NIV may reduce the risk of extubation failure over nasal CPAP and may be more beneficial than support provided by bi-level positive airway pressure. ${ }^{13}$

Although it would be easy to jump into adopting a new interface for providing noninvasive respiratory support, it is important to carefully review the information on any new device and/or modality to determine its safety profile before implementation. Given that the delivery of nasal CPAP or NIV is an off-label use with the RAM cannula, special care should be taken when using it in this scenario. There should be a $60-80 \%$ occlusion of the nares, however, in vitro lung model studies ${ }^{14,15}$ have shown mixed results in whether this amount of occlusion delivers the set pressures, with one study ${ }^{15}$ that showed pressures lower by approximately $30 \%$. This potential concern for decreased pressure transmission may be a factor in the higher 


\section{EDITORIALS}

pressures used in the post-RAM cannula era in the study presented. Despite these higher pressures, however, the authors did not find higher rates of bronchopulmonary dysplasia, in fact, they noted a trend toward lower rates of bronchopulmonary dysplasia and significantly fewer days that required respiratory support. ${ }^{1}$ This may indicate that they were able to safely overcome leaks and the resistance of the RAM cannula with the use of higher set pressures; however, this must always be done with extreme caution to avoid lung injury because reports are conflicting regarding pressure transmission via the RAM cannula.

Although the modalities for noninvasive respiratory support are numerous, I feel strongly that the mainstay of noninvasive respiratory support for the premature infant is nasal CPAP, given the constant distending pressure that maintains functional residual capacity. In addition, NIV may also have a role in preventing extubation failure in those patients who could not tolerate nasal CPAP. Weaning patients too soon to HFNC or to low-flow nasal cannula may ultimately result in longer respiratory support times because these modes may not provide the same consistent support as nasal CPAP. Given the current study and my own clinical experience, the RAM cannula may prove to be a promising way to deliver respiratory support without the concerns for nasal breakdown and patient discomfort noted with current interfaces. However, as with the implementation of any new device, I urge centers to take the time to educate providers, establish a protocol for use, and track their outcomes and critically evaluate the efficacy of the new device. In addition, special attention should be given to pressure delivery because there is the potential for underdelivering pressure with this interface.

Suma B Hoffman MD

Department of Pediatrics

University of Maryland School of Medicine Baltimore, Maryland

\section{REFERENCES}

1. Drescher GS, Hughes CW. Comparison of interfaces for the delivery of noninvasive respiratory support to low birth weight infants. Respir Care 2018;63(10):1197-1206.
2. Davis PG, Henderson-Smart DJ. Nasal continuous positive airways pressure immediately after extubation for preventing morbidity in preterm infants. Cochrane Database Syst Rev 2003(2): CD000143.

3. Subramaniam P, Henderson-Smart DJ, Davis PG. Prophylactic nasal continuous positive airways pressure for preventing morbidity and mortality in very preterm infants. Cochrane Database Syst Rev 2005(3):CD001243.

4. Morley CJ, Davis PG, Doyle LW, Brion LP, Hascoet JM, Carlin JB; COIN Trial Investigators. Nasal CPAP or intubation at birth for very preterm infants. N Engl J Med 2008;358(7):700-708.

5. Aly H, Milner JD, Patel K, El-Mohandes AA. Does the experience with the use of nasal continuous positive airway pressure improve over time in extremely low birth weight infants? Pediatrics 2004; 114(3):697-702.

6. Diblasi RM. Nasal continuous positive airway pressure (CPAP) for the respiratory care of the newborn infant. Respir Care 2009;54(9): 1209-1235.

7. Collins CL, Barfield C, Horne RS, Davis PG. A comparison of nasal trauma in preterm infants extubated to either heated humidified highflow nasal cannulae or nasal continuous positive airway pressure. Eur J Pediatr 2014;173(2):181-186.

8. Collins CL, Holberton JR, Barfield C, Davis PG. A randomized controlled trial to compare heated humidified high-flow nasal cannulae with nasal continuous positive airway pressure postextubation in premature infants. J Pediatr 2013;162(5):949-954.

9. Holleman-Duray D, Kaupie D, Weiss MG. Heated humidified highflow nasal cannula: use and a neonatal early extubation protocol. J Perinatol 2007;27(12):776-781.

10. Hoffman SB, Terrell N, Driscoll CH, Davis NL. Impact of high-flow nasal cannula use on neonatal respiratory support patterns and length of stay. Respir Care 2016;61(10):1299-1304.

11. Abdel-Hady H, Shouman B, Aly H. Early weaning from CPAP to high flow nasal cannula in preterm infants is associated with prolonged oxygen requirement: a randomized controlled trial. Early Hum Dev 2011;87(3):205-208.

12. Sasi A, Malhotra A. High flow nasal cannula for continuous positive airway pressure weaning in preterm neonates: A single-centre experience. J Paediatr Child Health 2015;51(2):199-203.

13. Lemyre B, Davis PG, De Paoli AG, Kirpalani H. Nasal intermittent positive pressure ventilation (NIPPV) versus nasal continuous positive airway pressure (NCPAP) for preterm neonates after extubation. Cochrane Database Syst Rev 2017;2:CD003212.

14. Iyer NP, Chatburn R. Evaluation of a nasal cannula in noninvasive ventilation using a lung simulator. Respir Care 2015;60(4): 508-512.

15. Gerdes JS, Sivieri EM, Abbasi S. Factors influencing delivered mean airway pressure during nasal CPAP with the RAM cannula. Pediatr Pulmonol 2016;51(1):60-69. 